\title{
Cambios en la partición de flujos de agua en el Chaco Árido al reemplazar bosques por pasturas
}

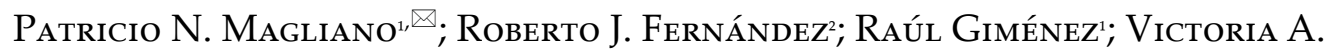 \\ Marchesin I ${ }^{13} ;$ Ricardo A. PÁeZ \& Esteban G. JobbágY ${ }^{1}$ \\ ${ }^{1}$ Grupo de Estudios Ambientales-IMASL, Universidad Nacional de San Luis-CONICET, Argentina. ${ }^{2}$ Cátedra de Ecología \\ e IFEVA, Facultad de Agronomía, Universidad de Buenos Aires-CONICET, Argentina. ${ }^{3}$ School of Plant Biology, The \\ University of Western Australia, Australia
}

\begin{abstract}
Resumen. La vegetación cumple un rol muy importante regulando los flujos de agua de los ecosistemas, en especial en regiones áridas y semiáridas donde la mayor parte de la precipitación se evapotranspira. En este trabajo se exploró cómo el reemplazo de los bosques secos nativos por pasturas, orientado a aumentar la productividad ganadera, afecta la partición de flujos de agua en las llanuras del Chaco Árido. Se caracterizó la distribución del tamaño y la intensidad de eventos de precipitación, y se midieron los flujos de intercepción directa, escurrimiento superficial, drenaje profundo y evaporación potencial en parcelas pareadas de bosques y pasturas en San Luis, Argentina. Las pasturas presentaron menor intercepción directa $(0.35 \pm 0.13 \mathrm{vs}$. $1.51 \pm 0.50$ $\mathrm{mm}$ /evento de precipitación), mayor escurrimiento superficial ( $28 \mathrm{vs.} 0 \%$ de escurrimiento en eventos grandes e intensos) y mayor evaporación potencial a nivel del suelo ( $7.0 \pm 0.9$ vs. $4.4 \pm 0.9 \mathrm{~mm} /$ día en el verano). El drenaje profundo resultó nulo en ambas coberturas, aunque bajo las pasturas se encontró un desplazamiento vertical de las sales del perfil hasta los $2 \mathrm{~m}$ de profundidad. Luego de cada evento de precipitación, las pasturas presentaron una distribución más homogénea de la humedad edáfica como producto de una menor redistribución de agua por el canopeo y la superficie del terreno. Estos resultados sugieren que el establecimiento de pasturas en sitios originalmente cubiertos por bosques reduciría la fracción de la precipitación potencialmente transpirable y aumentaría el riesgo potencial de erosión hídrica. La importancia de los flujos de redistribución sobre el balance hídrico y el funcionamiento ecohidrológico del sistema resalta la necesidad de caracterizar la dinámica del agua a escala de parche en paisajes llanos.
\end{abstract}

[Palabras clave: balance de agua, Chaco Seco, desmonte, intensificación, ganadería extensiva, cambio en el uso de la tierra]

\begin{abstract}
Aвstract: Changes in water fluxes partition in the Arid Chaco caused by the replacement of forest by pastures. Vegetation plays an important part in regulating water fluxes in ecosystems, specially in dry regions where a large fraction of precipitation is evapotranspirated. Here, we assessed how the replacement of native dry forests by pastures, with the aim of increasing livestock production, affects water flux partition in the Arid Chaco plains. We characterized the size and intensity distribution of precipitation events, and we measured canopy interception, runoff, deep drainage and potential evaporation in paired stands of dry forests and pastures in San Luis, Argentina. Pastures presented lower canopy interception ( $0.35 \pm 0.13$ vs. $1.51 \pm 0.50 \mathrm{~mm} /$ precipitation event), higher runoff ( $\sim 28$ vs. $0 \%$ runoff in large and intense precipitation events) and higher potential evaporation $(7.0 \pm 0.9$ vs. $4.4 \pm 0.9 \mathrm{~mm} /$ day in summer). Deep drainage was nil in both cover types, but a $2 \mathrm{~m}$ downward salt displacement was found under pastures. After each precipitation event, soil moisture horizontal distribution was more homogenous in pastures, as a result of less canopy and soil-surface water redistribution at the patch scale. Our results suggest that the establishment of pastures in sites previously covered by forests would reduce the proportion of precipitation potentially transpired by vegetation and increase the potential risk of water erosion. We highlight the need for a better understanding of water dynamics at the patch scale in these plain landscapes, due to the relevance that water redistribution processes have in explaining water balance and the ecohydrology of the system.
\end{abstract}

[Keywords: water balance, Dry Chaco, clearing, intensification, extensive livestock production, land use/land cover change]

Editor asociado: Fabián Scholz

$\triangle$ pnmagliano@gmail.com
Recibido: 2 de septiembre de 2015

Aceptado: 15 de abril de 2016 


\section{INTRODUCCIÓN}

El funcionamiento de los ecosistemas terrestres secos depende de la fracción de la precipitación que ingresa en el suelo y puede ser absorbida por la vegetación (Noy-Meir 1973; Austin et al. 2004). Las regiones áridas y semiáridas, donde el agua disponible es la limitante principal para la productividad primaria neta (PPN), ocupan $\sim 45 \%$ de la superficie continental del planeta (Newman et al. 2006; Schimel 2010). En el balance hídrico de la mayoría de estas regiones, $\sim 95 \%$ de las precipitaciones vuelve a la atmósfera como transpiración vegetal y evaporación directa (Schwinning et al. 2004; Schlesinger and Jasechko 2014), lo que pone de manifiesto el papel clave que cumple la vegetación al regular las salidas de vapor de agua del sistema. La intercepción directa (y la consecuente pérdida de agua por evaporación desde el canopeo), el escurrimiento superficial y el drenaje profundo suelen representar una fracción pequeña del balance hídrico (Levia and Frost 2003; Carlyle-Moses 2004; Scanlon et al. 2005; Yaseef et al. 2010).

La partición de los flujos de agua de los ecosistemas está determinada por las interacciones entre la precipitación, la vegetación y el suelo (Chapin et al. 2002; Knapp et al. 2002; Nicholson 2011) (Figura 1). La precipitación ejerce un control fuerte sobre la transpiración vegetal a través de su magnitud anual: a mayor precipitación anual, mayor PPN anual (Sala et al. 1988; Huxman et al. 2004;

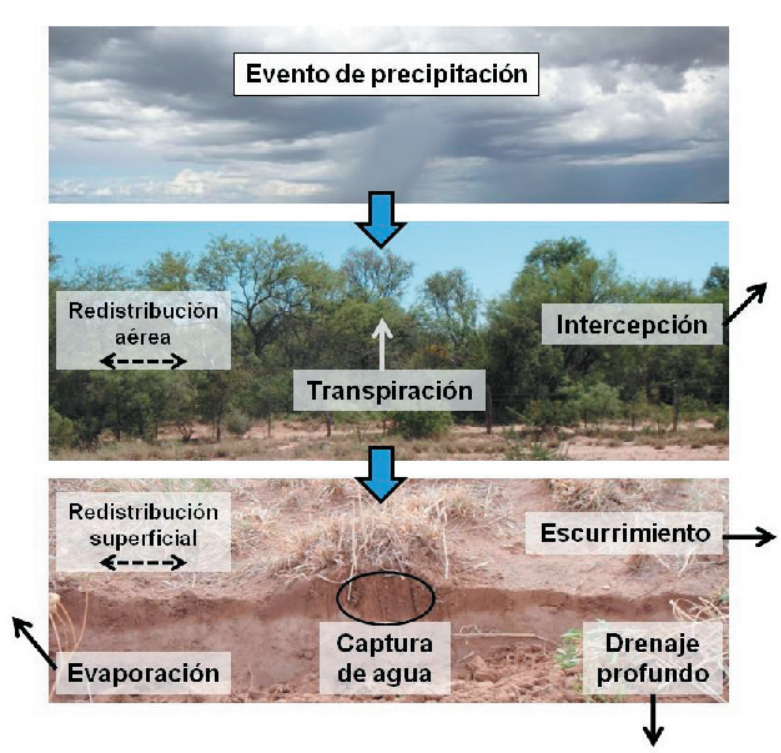

Del Grosso et al. 2008) y de la distribución de los eventos (Knapp et al. 2002; Yahdjian and Sala 2006; Magliano et al. 2015b). Por ejemplo, para una dada cantidad de precipitación anual, una distribución caracterizada por eventos pequeños muy frecuentes tenderá a favorecer las pérdidas por evaporación. Una distribución carcterizada por eventos grandes y poco frecuentes favorecerá la transpiración vegetal, el escurrimiento superficial y el drenaje profundo (Harper et al. 2005; Hao et al. 2013). La vegetación afecta la partición de flujos de agua en forma directa. Los sitios más vegetados se distinguen por tener mayor transpiración y menor escurrimiento, drenaje profundo y evaporación que sitios menos vegetados (Newman et al. 2006; Schlesinger and Jasechko 2014). Sin embargo, también puede actuar en forma indirecta al afectar la redistribución espacial del agua del suelo, concentrándola en parches vegetados y favoreciendo la transpiración por sobre los demás flujos (Ludwig et al. 2005; Bautista et al. 2007; Magliano et al. 2015a). El microrelieve, la textura y la estructura del suelo generan cambios en la partición entre infiltración y escurrimiento superficial en la escala de parche $\left(0.25 \mathrm{~m}^{2}\right)$; lo cual incide sobre la partición evaporación/ transpiración (E/T) en la escala de parcela (1 ha) (Reid et al. 1999; Caldwell et al. 2008; Caldwell et al. 2012). Por estos motivos, cuando la vegetación es reemplazada se alteran las interacciones precipitación-vegetación-suelo y, por ende, la partición de flujos de agua del ecosistema.

Figura 1. Esquema conceptual de la partición de los flujos de agua de un evento de precipitación. La foto central corresponde a un bosque seco característico del Chaco Árido, y la inferior a un corte en el perfil del suelo que muestra el frente de mojado correspondiente a un evento de precipitación de $8 \mathrm{~mm}$ (foto tomada 24 horas después de ocurrido el evento). Los flujos de agua se encuentran representados por las flechas de trazos continuos. Las flechas negras representan los flujos de pérdida de agua (intercepción, escurrimiento, drenaje profundo y evaporación). La flecha gris representa la transpiración vegetal (flujo productivo). Los procesos de redistribución de agua se encuentran representados por las flechas de trazos y la captura de agua (lámina de agua infiltrada) por el óvalo.

Figure 1. Conceptual model of water fluxes partition after a precipitation event. The central photo corresponds to a typical dry forest of Arid Chaco, and the photo at the bottom shows the wetting front after an $8 \mathrm{~mm}$ precipitation event (24 hours after the event). Water fluxes are represented by solid arrows. Black arrows represent water losses (interception, runoff, deep drainage, evaporation). The grey arrow represents transpiration (productive flux). Water redistribution processes are represented by stripped arrows and water capture (water infiltrated depth) by the oval. 
Los reemplazos bruscos de vegetación pueden modificar el balance de agua en forma significativa (Farley et al. 2005; Nosetto et al. 2012; Marchesini et al. 2015). Por ejemplo, el reemplazo de bosques por cultivos reduce la transpiración vegetal del ecosistema, que en muchos casos se puede traducir en aumentos en los flujos de escurrimiento superficial, drenaje profundo y evaporación directa (Connolly 1998; Foley et al. 2005; Scanlon et al. 2005; Bondeau et al. 2007). El aumento del escurrimiento superficial incrementa los riesgos de erosión hídrica y/o inundaciones, pero también puede aprovecharse si se lo logra cosechar en represas (tajamares) para utilizarlo como fuente de agua líquida para bebida animal (Oweis and Hachum 2009; Magliano et al. 2015c). En el largo plazo, el aumento en el drenaje profundo en paisajes de llanura puede generar ascensos del nivel freático, salinización superficial y pérdidas de la capacidad productiva del ecosistema (Eberbach 2003; Scanlon et al. 2005; Jobbágy et al. 2008). El aumento en la evaporación directa reduce la eficiencia en el uso del agua del ecosistema (menor PPN con la misma precipitación) (Huxman et al. 2005; Newman et al. 2006). Todo esto pone en evidencia la importancia que tiene la vegetación en la partición de flujos de agua en sistemas áridos y semiáridos, en especial en aquellas regiones donde los cambios en el uso del suelo avanzan rápidamente, como ocurre en el Chaco Árido (Baldi et al. 2015).

El Chaco Árido ocupa una planicie sedimentaria de 10 millones de ha, cubierta por bosques secos nativos dedicados en su mayoría a la ganadería bovina extensiva y, en menor medida, a la caprina (Rueda et al. 2013). Estos bosques están siendo reemplazados por pasturas de Eragrostis curvula (pasto llorón), Cenchrus ciliaris (pasto búfalo) y Panicum maximum (gatton panic) con el objetivo de aumentar la productividad ganadera (Kunst et al. 2003; Anriquez et al. 2005; Blanco et al. 2005b; Kunst et al. 2012). Este reemplazo brusco y masivo de la vegetación leñosa nativa puede generar grandes cambios en los flujos de agua, en particular en los sitios más intensamente transformados. En este trabajo se integra un conjunto de observaciones y mediciones realizadas sobre bosques secos nativos de la provincia de San Luis (Argentina) y sobre las pasturas que los han reemplazado, con el fin de realizar un aporte cuantitativo al conocimiento de los efectos de este reemplazo de vegetación sobre el balance de agua de estos ecosistemas. Se combina el análisis de la distribución de tamaño e intensidad de eventos de precipitación con mediciones directas de flujos y volúmenes de agua en parcelas pareadas de bosques y pasturas.

\section{MATERIALES Y MÉTODOS}

\section{Sitio de estudio}

El Chaco Árido corresponde a la porción más austral del Chaco Seco, que representa la planicie sedimentaria dominada por bosques secos más grande de América (Morello and Toledo 1959; Morello and Adámoli 1974; Jobbágy et al. 2008). La pendiente regional del terreno es $\sim 1 \%$, con suelos derivados de la acumulación masiva de sedimentos eólicos y en menor medida aluviales durante el Cuaternario (Iriondo 1993; Pennington et al. 2000). Los suelos son Torriortentes Típicos, franco-arenosos ( $55 \%$ de arena, $15 \%$ de arcilla), con $1.5 \%$ de materia orgánica en los primeros $10 \mathrm{~cm}$, débilmente estructurados, y con una proporción escasa de grava en todo el perfil (Peña Zubiate et al. 1998). La secuencia típica de los horizontes edáficos es A $(0-20 \mathrm{~cm})$, AC1 $(20-40 \mathrm{~cm})$, AC2 $(40-65 \mathrm{~cm}), C(65-130$ $\mathrm{cm})$ (datos suministrados por Ser Beef S.A.). La precipitación media es de $430 \mathrm{~mm} /$ año, con un promedio de 43 eventos/año, concentrados entre septiembre y marzo (Magliano et al. 2015b). La napa freática se encuentra a $>30$ $\mathrm{m}$ de profundidad, por lo que es difícil que exista algún tipo de interacción entre la napa y la vegetación (Marchesini, comunicación personal). La vegetación nativa hace un uso exhaustivo del agua de lluvia debido a la combinación de especies de sistemas radicales profundos que exploran todo el perfil del suelo hasta más de $6 \mathrm{~m}$, baja pendiente regional y escasa precipitación anual. Por este motivo, el drenaje profundo es casi inexistente en el sitio de estudio (Marchesini et al. 2013), e incluso en la región vecina (Espinal), que dispone de $\sim 50 \%$ más de precipitación anual $(600 \mathrm{~mm} /$ año) (Santoni et al. 2010). Las mediciones que se presentan en este trabajo se realizaron en establecimientos ganaderos del Chaco Árido, en parcelas pareadas cubiertas por bosques secos y pasturas implantadas. El bosque seco ( $\sim 7 \mathrm{~m}$ de altura) está dominado por Prosopis flexuosa(algarrobo), Aspidosperma quebracho blanco (quebracho blanco) y Larrea divaricata (jarilla). Las pasturas se implantaron en reemplazo del bosque nativo en el año 1995. La especie elegida fue Eragrostis curvula, 
reemplazada en el 2012 por Cenchrus ciliaris (información brindada por la empresa Ser Beef S.A.).

\section{Diseño de los muestreos}

Los muestreos se realizaron en el extremo sur del Chaco Árido $\left(33.5^{\circ} \mathrm{S} ; 66.5^{\circ} \mathrm{O}\right)$ durante el período 2011-2014. Se empleó un diseño de parcelas pareadas de bosque/pastura de $\sim 1$ ha cada una $(n=3)$, para evitar diferencias edafoclimáticas ajenas a las coberturas vegetales. Los tres pares de parcelas de bosque/pastura seleccionados son representativos de los bosques y pasturas de la región en lo que respecta a características de la vegetación y a la dinámica espacial y temporal del agua del suelo de 0 a $3 \mathrm{~m}$ de profundidad (Marchesini 2011). En cada par se plantearon 2 transectas de muestreo (una en la parcela de bosque y una en la parcela de pastura) de $36 \mathrm{~m}$ de largo cada una, que incluyeron 18 parches de $50 \times 50 \mathrm{~cm}$, espaciados de forma sistemática cada $2 \mathrm{~m}$. Las transectas se orientaron en direcciones diferentes (este-oeste, norte-sur y noroeste-sudeste) para evitar cualquier sesgo introducido por la escasa pendiente regional. Sobre estas transectas se midieron los flujos de intercepción directa, captura de agua (a partir de la cual se estimó el escurrimiento superficial) y evaporación potencial. Dentro de las parcelas, pero fuera de las transectas, se realizaron mediciones de profundidad del frente de mojado luego de un evento de precipitación y de drenaje profundo.

\section{Mediciones}

La precipitación se cuantificó con una estación meteorológica automática instalada en una de las pasturas, que registró la precipitación en intervalos de 20 minutos (pluviómetro TR-525, Campbell Scientific; 2011-2014). Estos datos fueron previamente corroborados y validados con mediciones de seis pluviómetros manuales ubicados en zonas despejadas de canopeo dentro de cada parcela, con el fin de evitar cualquier sesgo derivado de la variabilidad espacial entre parcelas. El tamaño de los eventos de precipitación fue determinado como la suma de la lámina de todos los intervalos de 20 minutos que no estuvieron separados entre sí por más de $24 \mathrm{~h}$. La intensidad de los eventos fue determinada como el promedio de las intensidades registradas cada 20 minutos ponderadas por la lámina registrada en ese intervalo.
La intercepción directa se cuantificó como la diferencia entre la precipitación registrada por la estación meteorológica automática y la lámina colectada por pluviómetros manuales (cilindros de plástico de $10 \mathrm{~cm}$ de diámetro y 18 cm de altura) ubicados a nivel del suelo, en cada posición de las transectas. Los pluviómetros se revisaron 3-6 h luego de cada evento de precipitación (11 eventos muestreados entre 2012 y 2013); se registró la lámina precipitada y se vació su contenido hasta el evento siguiente. Si bien la metodología empleada no permite separar el flujo de intercepción directa del flujo de escurrimiento cortical ("stem flow"), los datos aquí presentados, mediciones de escurrimiento cortical en el Chaco Árido (Pedro Namur, comunicación personal) y resultados de la literatura en otras regiones áridas con especies vegetales similares (Martinez-Meza and Whitford 1996; Reynolds et al. 1999; Llorens and Domingo 2007) permiten asumir como despreciable el flujo cortical a escala de parcela.

La captura de agua, complemento del escurrimiento superficial, se define como el cociente entre la lámina infiltrada en el suelo 24 h después de finalizado un evento de precipitación y la lámina precipitada (ver debajo). En cada parche de las transectas se calculó la captura de agua para 4 eventos de precipitación de similar tamaño (desde 36 hasta $51 \mathrm{~mm} /$ evento), pero que representaron un rango amplio de intensidades (desde 9 hasta $34 \mathrm{~mm} / \mathrm{h}$ ). Los 4 eventos muestreados estuvieron precedidos por períodos de más de 15 días sin precipitación, lo que aseguró un nivel bajo de humedad volumétrica del suelo (HV), similar para todos los casos. La HV del día posterior a cada evento de precipitación fue medida con un sensor tipo TDR (time-domain reflectometry; modelo Theta-probe, Delta-T Devices), en intervalos de $10 \mathrm{~cm}$ de profundidad desde la superficie del suelo hasta el final del frente de mojado. La HV del suelo seco fue determinada luego de un largo período seco una única vez en agosto de 2013, llevando el muestreo hasta $1 \mathrm{~m}$ de profundidad. La captura de agua (\%) de cada parche se calculó como el cociente entre la lámina de agua infiltrada en el suelo luego de un evento de precipitación (mm) y la lámina de agua precipitada (mm). La lámina de agua infiltrada en el suelo se determinó como la diferencia entre la HV 24 h después del evento de precipitación y la HV del suelo seco (previa al evento), multiplicada por el frente de mojado alcanzado en el perfil del 
suelo (mm) (Hillel 1998). La diferencia entre la lámina precipitada ( $\mathrm{mm}$ ) y la captura de agua promedio de cada parcela $(\mathrm{mm})$ representó el escurrimiento superficial más la intercepción, la cual fue descontada para aislar el flujo de escurrimiento.

Se midió el frente de mojado de un evento de $54 \mathrm{~mm}$ de magnitud y $16 \mathrm{~mm} / \mathrm{h}$ de intensidad (febrero 2013) que tuvo lugar luego de un período seco de 15 días sin precipitaciones. Las mediciones se realizaron en parches de $50 \times 50 \mathrm{~cm}$ con cobertura vegetal contrastante (parches muy vegetados y parches sin vegetación), tanto en el bosque como en las pasturas. Se consideraron parches muy vegetados a aquellos con tres estratos de canopeo (herbáceo, arbustivo y arbóreo) y broza sobre la superficie del suelo en el caso del bosque, y al menos un ejemplar adulto de Cenchrus ciliaris en el caso de las pasturas. Se consideraron parches sin vegetación a aquellos desprovistos de cobertura aérea y con suelo desnudo en superficie, tanto para el bosque como para las pasturas. De este modo, se plantearon cuatro situaciones: bosquevegetado, bosque-sin vegetación, pasturavegetada, pastura-sin vegetación. Se registró el frente de mojado en 5 parches distribuidos al azar, correspondientes a cada una de estas situaciones en cada parcela.

El drenaje profundo se caracterizó a partir del patrón de distribución de cloruros $\left(\mathrm{Cl}^{-}\right)$en el perfil del suelo de las parcelas de bosque y pastura (Scanlon 1991; Santoni et al. 2010). El cloruro es un ión muy soluble que se incorpora al suelo casi exclusivamente por deposición atmosférica húmeda (disuelto en la precipitación) o seca (en forma de partículas), con mínima contribución del material parental. Al ser poco absorbido por las plantas, es un marcador conservativo que se pierde del sistema principalmente a través del drenaje profundo, por lo que se lo usa como indicador de este flujo en ambientes áridos, donde su acumulación en el suelo es grande y sus pérdidas ínfimas (Scanlon et al. 2002). En cada parcela de bosque y de pastura se realizó una perforación de muestreo de 6 $\mathrm{m}$ de profundidad, con barreno manual de 10 $\mathrm{cm}$ de diámetro $(\mathrm{n}=3)$. Se tomaron muestras de suelo cada $25 \mathrm{~cm}$ desde la superficie hasta $1 \mathrm{~m}$ de profundidad y cada $50 \mathrm{~cm}$ entre 1 y $6 \mathrm{~m}$ de profundidad. Para analizar la concentración de $\mathrm{Cl}^{-}$en las muestras se efectuaron extractos 2:1 de agua destilada: suelo, y se utilizó un electrodo selectivo de estado sólido (ORION 94-17, Thermo Electron Corporation).
La evaporación potencial hace referencia a la lámina potencialmente evaporable a nivel del suelo. Para cuantificar su magnitud bajo cada tipo de cobertura se instalaron micro-lisímetros en los parches situados a lo largo de las transectas. Los micro-lisímetros constaron de cilindros plásticos de $10 \mathrm{~cm}$ de diámetro y $18 \mathrm{~cm}$ de altura, rellenos con $1 \mathrm{~kg}$ de suelo del horizonte superficial $(0-10 \mathrm{~cm})$ del sitio de estudio, previamente tamizado y homogenizado, dispuestos dentro de tubos de PVC enterrados a nivel del suelo. Se colocó un micro-lisímetro en cada parche de las pasturas, y dos micro-lisímetros por parche de bosque: uno con suelo desnudo y otro con $3 \mathrm{~cm}$ de espesor de broza recolectada en las inmediaciones del punto. Esta manipulación buscó separar el efecto de la sombra del canopeo del efecto de la broza de suelo sobre la evaporación potencial en el bosque. En cada micro-lisímetro se aplicó una lámina de agua de $28 \mathrm{~mm}$ para llevar el suelo a capacidad de campo, según determinaciones de laboratorio basadas en el método desarrollado por Colman (1947) (Colman 1947; Hillel 1998). En el comienzo del ensayo se registró el peso inicial de cada micro-lisímetro (09/ 02/2012, 9:00 am), y el peso luego de $24 \mathrm{~h}$. La diferencia de peso representó la lámina potencialmente evaporable ( $\mathrm{mm} /$ día). Las condiciones meteorológicas al momento del ensayo (radiación incidente $=198.3 \mathrm{~W} / \mathrm{m}^{2}$, temperatura $=24.3{ }^{\circ} \mathrm{C}$, humedad relativa $=45 \%$, velocidad de viento $=5.8 \mathrm{~m} / \mathrm{s}$ y $E T 0=7 \mathrm{~mm} /$ día, según FAO Penman-Monteith [Allen et al. 1998]) se corresponden con valores diarios promedio de la estación estival en que ocurren las principales lluvias.

\section{Análisis estadísticos}

Los análisis empleados para detectar diferencias en los flujos/volúmenes de agua entre las coberturas de bosque y pastura variaron según las características del muestreo. Para las mediciones en que se incluyeron varios eventos de precipitación (intercepción directa y captura de agua) se realizaron análisis de varianza bi-factoriales (ANOVA de dos vías), donde el tipo de cobertura representó el factor principal ( 2 niveles: bosque y pastura) y los eventos de lluvia el factor secundario (11 y 4 eventos para intercepción directa y captura de agua, respectivamente). También se utilizó un análisis de varianza bi-factorial para analizar la interacción entre el tipo de cobertura vegetal (factor principal con 2 niveles: bosque y pastura) y la densidad de la misma 
(factor secundario con 2 niveles: vegetado y sin vegetación) sobre la profundidad del frente de mojado. La comparación de la concentración de cloruros en el perfil del suelo entre ambas coberturas para caracterizar el drenaje profundo se realizó mediante pruebas de $t$ apareadas $(n=3)$ para cada estrato de profundidad. Las comparaciones de evaporación potencial se realizaron mediante un análisis de varianza unifactorial $(n=3)$ con tres niveles de tipo de cobertura: bosque con suelo desnudo, bosque con broza agregada y pastura, y pruebas de $\mathrm{t}$ para comparaciones más específicas (e.g., pastura vs. valores de bosque>percentil 95). Además, se emplearon modelos de regresión lineal para establecer la relación entre el tamaño y la intensidad de los eventos de precipitación registrados por la estación meteorológica entre fines de 2011 e inicios de 2014 ( $n=80)$ y entre la intensidad de los eventos y la variabilidad espacial en la captura de agua en ambas coberturas $(n=4$, en cada caso).

\section{Resultados}

Tanto el tamaño como la intensidad de los eventos de precipitación presentaron una distribución asimétrica de sus valores; se encontraron unos pocos eventos desproporcionadamente grandes/muy intensos, y muchos eventos pequeños / poco intensos (Figura 2). Sólo el evento de precipitación más grande representó el 15\% de la precipitación media anual. La sumatoria de los dos y de los seis eventos de precipitación más grandes explicó 25 y el $50 \%$ de la precipitación media anual, respectivamente, mientras que la sumatoria de los 15 eventos más pequeños sólo explicó 2\% (Figura 2A). Si bien se encontró una relación positiva y significativa entre el tamaño y la intensidad de los eventos de precipitación $\left(\mathrm{R}^{2}=0.45 ; P<0.01\right)$ (Figura 2C), cabe destacar que los eventos más intensos no necesariamente corresponden a los eventos más grandes.

La intercepción directa promedio de las parcelas varió según el tipo de cobertura $(P<0.0001)$, pero no entre eventos de precipitación (tamaño: $P>0.05$; intensidad: $P>0.05)$. El bosque presentó una intercepción de $1.51 \pm 0.50 \mathrm{~mm}$ /evento (media y desvío estándar), mientras que la intercepción de las pasturas fue de $0.35 \pm 0.13 \mathrm{~mm} /$ evento (media y desvío estándar).

La captura de agua a escala de parcela varió tanto por efecto del tipo de cobertura (bosque, pastura: $P<0.05$ ) como del evento
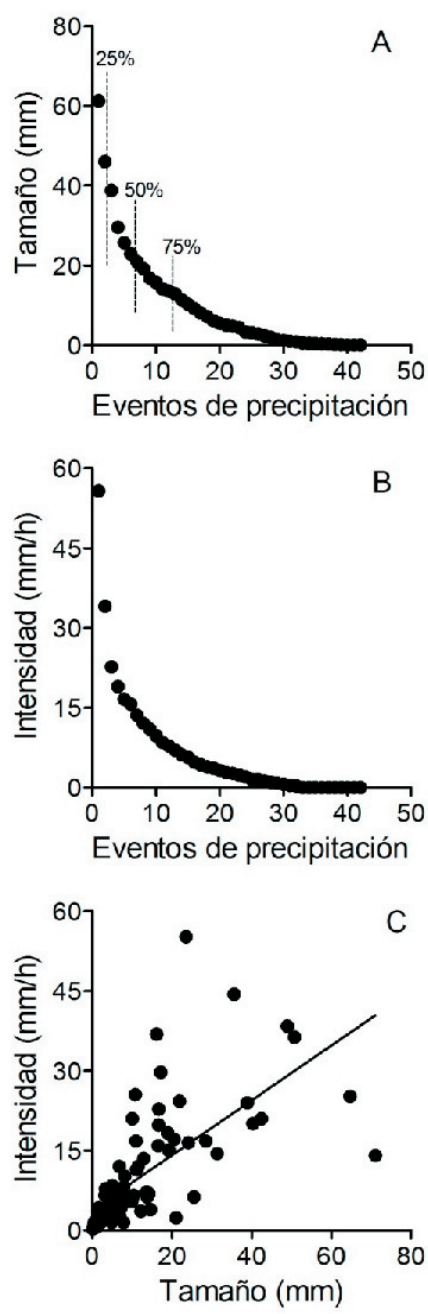

Figura 2. Distribución de tamaño (A) e intensidad (B) de los eventos de precipitación ocurridos en un año típico (promedio 2011-2014) en la región de estudio (430 $\mathrm{mm} / \mathrm{año}, 43$ eventos/año). Los marcadores circulares representan eventos individuales, ordenados de mayor a menor. Los porcentajes en A indican los eventos que suman 25,50 y $75 \%$, respectivamente, de la precipitación media anual. (C) Relación entre la intensidad y el tamaño de los eventos de precipitación ocurridos en el período 2011-2014 (y=0.52x+3.84; $\left.\mathrm{R}^{2}=0.45 ; P<0.01\right)$.

Figure 2. Size (A) and intensity (B) precipitation event distribution of a typical year (average 2011-2014) at the study region $(430 \mathrm{~mm} /$ year, 43 events/year). Circular markers represent isolated precipitation events, ordered from the highest to the lowest. The percentages in A show the events that accounted for 25,50 and $75 \%$ of mean annual precipitation. (C) Event intensity as a function of event size for all the events occurred during the study period 2011-2014 $\left(\mathrm{y}=0.52 \mathrm{x}+3.84 ; \mathrm{R}^{2}=0.45 ; P<0.01\right)$

de precipitación (tamaño: $P<0.05$; intensidad: $P<0.01)$. En tres de los cuatro eventos muestreados, la captura fue similar y cercana al $100 \%$ en ambas coberturas; sólo en el evento más intenso $(34 \mathrm{~mm} / \mathrm{h}$, percentil 95 en intensidad) la pastura presentó una captura significativamente inferior a la del bosque 


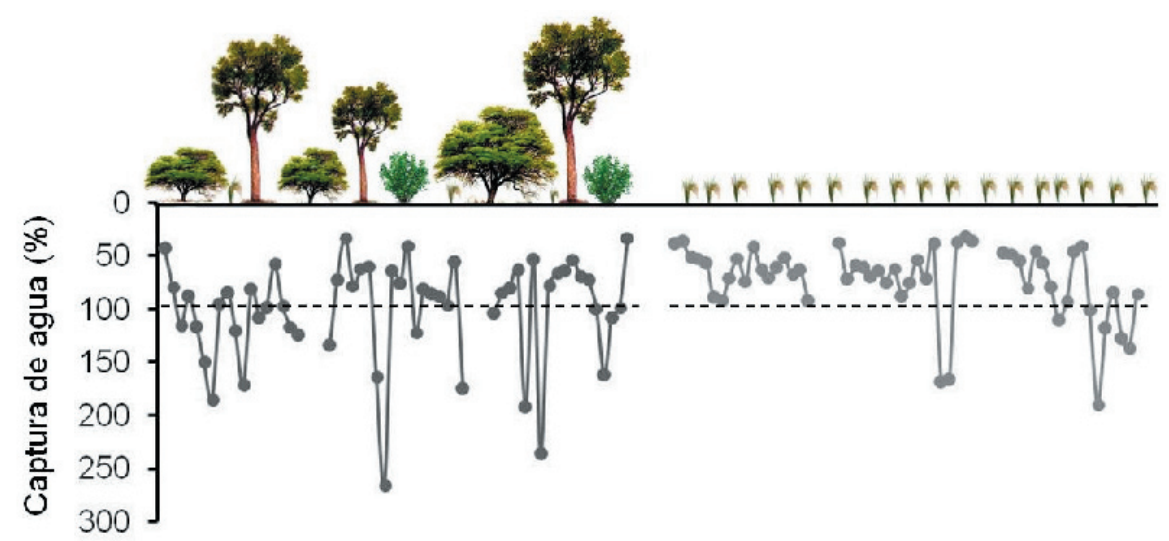

Figura 3. Captura de agua del evento de precipitación más intenso $(34 \mathrm{~mm} / \mathrm{h})$ muestreado a lo largo de las transectas $(\mathrm{n}=3$ transectas $\times 18$ parches $=54$ datos), para el bosque (izquierda) y la pastura (derecha). Cada transecta tuvo una longitud de $36 \mathrm{~m}$. La línea de trazos indica 100\% de captura de agua; parches $<100 \%$ recibieron menos agua que la precipitada (fuentes); parches $>100 \%$ recibieron más agua que la precipitada (destinos o sumideros). La captura de agua promedio de las tres transectas fue $99.6 \pm 48.7 \%$ (media y desvío estándar) en el bosque y $72.3 \pm 34.4 \%$ (media y desvío estándar) en la pastura.

Figure 3. Water capture after the highest intensity precipitation event $(34 \mathrm{~mm} / \mathrm{h})$ sampled along $36 \mathrm{~m}$-long transects $(\mathrm{n}=3$ transects $x 18$ patches $=54$ ), for forest (left) and pasture (right) covers. Each transect was $36 \mathrm{~m}$ long. Broken line indicates $100 \%$ water capture; patches $<100 \%$ received less water than precipitation (sources) and patches $>100 \%$ received more water than precipitation (sinks). Mean water capture of three transects was $99.6 \pm 48.7 \%$ (mean and standard deviation) in forest and $72.3 \pm 34.4 \%$ (mean and standard deviation) in pasture.

$(P<0.01)$. En este evento, la captura de agua de la pastura fue $72.3 \pm 34.4 \%$ (media y desvío estándar), mientras que la captura de agua del bosque fue $99.6 \pm 48.7 \%$ (media y desvío estándar). Esto implicó que, tras un evento de precipitación muy intenso, las parcelas de bosque tuvieron un escurrimiento superficial prácticamente nulo, mientras que las parcelas de pastura perdieron en promedio $27.7 \%$ de la lámina precipitada por escurrimiento superficial. A escala de parche se encontró, para ambos tipos de cobertura, una asociación lineal y positiva entre la intensidad de los eventos de precipitación y el coeficiente de variación espacial de la captura de agua $\left(P<0.01 ; \mathrm{n}=4\right.$ eventos; $\mathrm{R}^{2}=0.88$ y 0.66 , en el bosque y la pastura, respectivamente). La heterogeneidad espacial de la captura de agua resultó mayor en el bosque (Figura 3 y 4). Por un lado, el bosque presentó parches con valores de captura de agua más extremos que los de las pasturas (Figura 3). Por otro lado, los resultados de las mediciones del frente de mojado de un evento de precipitación de 54 $\mathrm{mm}$ de magnitud y $16 \mathrm{~mm} / \mathrm{h}$ de intensidad mostraron que, tanto para el bosque como para la pastura, los parches vegetados tuvieron una mayor profundidad del frente de mojado que los no vegetados $(P<0.01$; en bosque y pastura), siendo dicho patrón más contrastante en el bosque (Figura 4).

El drenaje profundo fue nulo tanto en las parcelas de bosque como en las parcelas de pastura, dado que se registró una cantidad

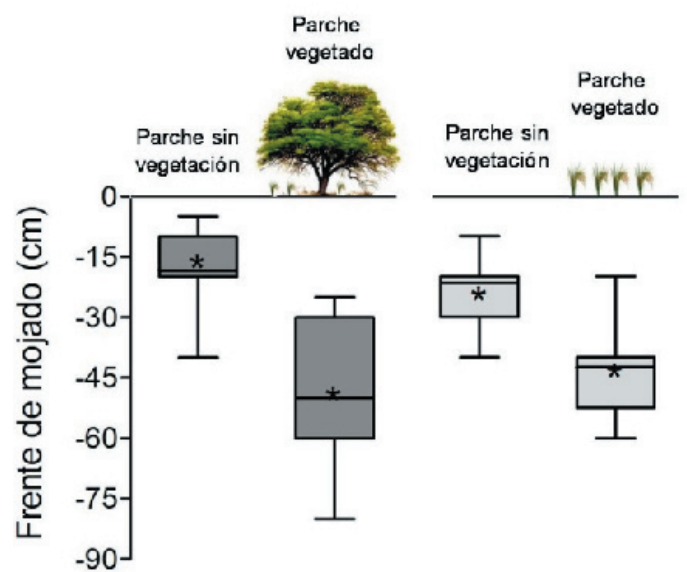

Figura 4. Frente de mojado para parches muy vegetados y sin vegetación luego de un evento de precipitación de $54 \mathrm{~mm}$ (intensidad: $16 \mathrm{~mm} / \mathrm{h}$ ), en el bosque (izquierda) y la pastura (derecha). Las cajas representan los cuartiles 1, 2 y 3; las líneas, el máximo y el mínimo; los asteriscos, la media para cada cobertura.

Figure 4. Wetting front depth for contrasting vegetation patches (full and not vegetated) after a $54 \mathrm{~mm}$ precipitation event (intensity: $16 \mathrm{~mm} / \mathrm{h}$ ), for forest (left) and pasture (right). Whisker plots show maximum, first quartile, median, third quartile, and minimum values for 54 patches; asterisks indicate the mean value for each cover.

importante de cloruros en la zona de exploración radical de ambas coberturas (hasta $\sim 2.5 \mathrm{~m}$ en la pastura y mayor en el bosque) (Figura 5). Sin embargo, la pastura presentó significativamente $(P<0.01)$ menor cantidad cloruros desde los $0.75 \mathrm{~m}$ hasta los $2 \mathrm{~m}$ de profundidad, lo que sugiere un lavado parcial de las sales del perfil respecto del bosque como 


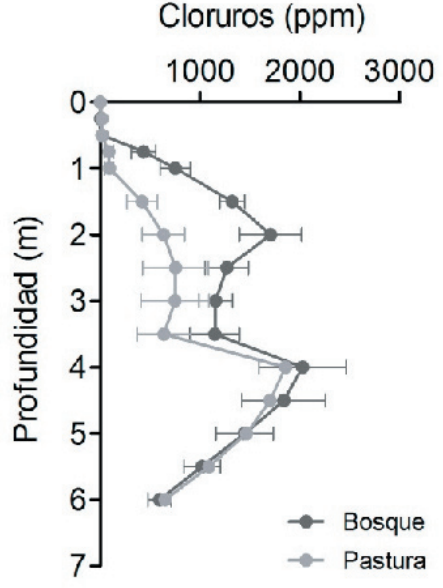

Figura 5. Perfiles de cloruros $\left(\mathrm{mg} \mathrm{Cl}^{-} / \mathrm{kg}\right.$ suelo) para el bosque y la pastura (de 20 años de antigüedad). Cada marcador es el promedio de 3 pozos de $6 \mathrm{~m}$ de profundidad. Las barras horizontales indican el desvío estándar.

Figure 5. Chloride concentration profiles ( $\mathrm{mg} \mathrm{Cl}^{-} / \mathrm{kg}$ soil) for forest and pasture. Each marker represents the mean of three 6-meter depth boreholes. Horizontal bars indicate the standard deviation of the mean.

consecuencia de una mayor percolación de agua hacia los estratos más profundos de la zona de exploración radical.

La evaporación potencial varió significativamente entre coberturas $(P<0.01)$. La evaporación potencial de la pastura fue $7.0 \pm 0.9 \mathrm{~mm} /$ día (media y desvío estándar), la del bosque con suelo desnudo fue $4.4 \pm 0.9$ $\mathrm{mm}$ /día (media y desvío estándar), y la del bosque con broza fue $1.2 \pm 0.3 \mathrm{~mm} /$ día (media y desvío estándar). En los parches de bosque con suelo desnudo, los valores de evaporación potencial más altos (percentil 95) no difirieron significativamente de la evaporación potencial promedio de los parches de pastura $(P=0.46)$; mientras que los valores más bajos (percentil 5) resultaron significativamente mayores al promedio de los parches con broza $(P<0.01)$.

\section{DisCUSIÓN}

En este trabajo se exploró cómo el reemplazo de bosques secos nativos por pasturas, orientado a aumentar la productividad ganadera, afectó la partición de flujos de agua en ecosistemas del Chaco Árido. Por un lado, se encontró una disminución en la intercepción directa y aumentos en el escurrimiento superficial y la evaporación potencial a nivel del suelo. Por otro lado, el lavado parcial de los cloruros del suelo en la zona de exploración radical de las pasturas indica la ocurrencia de flujos descendentes de agua que no se tradujeron aún en pérdidas por drenaje profundo, aunque sugieren una menor capacidad de utilizar el agua infiltrada respecto de los bosques. Las pasturas presentaron una captura de agua de lluvia espacialmente más homogénea que el bosque, producto de una menor redistribución de agua por el canopeo y la superficie del suelo. Esto sugiere que las pasturas, luego de 20 años de permanencia, reducen la heterogeneidad propia del bosque y conducen al sistema a una situación más similar a la de un pastizal natural (Kleb and Wilson 1997; Breshears and Barnes 1999; Wang et al. 2010). Si bien las mediciones no estuvieron orientadas a cuantificar el balance de agua en ambas coberturas, las mismas sugieren que la fracción de la precipitación potencialmente transpirable resultaría menor en las pasturas como resultado de las mayores pérdidas por escurrimiento y evaporación directa. Estos resultados evidencian la necesidad de abordar al sistema desde escalas más finas (parche) para entender algunos procesos que afectan a la partición de flujos de agua de la parcela, como por ejemplo la redistribución de agua entre parches y la heterogeneidad que esto genera en la distribución de humedad de los perfiles de suelo.

Desde la perspectiva de la maximización de la productividad primaria neta bajo condiciones de semiaridez, es deseable incrementar la transpiración vegetal y reducir el resto de los flujos (Noy-Meir 1973; Newman et al. 2006). El drenaje profundo resultó nulo en ambas coberturas (Figura 5). Esto sugiere que el bosque utiliza exhaustivamente el agua de las precipitaciones, algo ya observado en muchos sitios de la región (Santoni et al. 2010), y que su reemplazo por pasturas tiene poco efecto sobre esta característica, a diferencia de lo observado con el uso agrícola de estos ambientes (Contreras et al. 2013). El escaso desplazamiento vertical del frente de cloruros observado en las pasturas es similar a lo observado en bosques disturbados por "rolados" (Marchesini et al. 2013), y actualmente no representaría un riesgo de salinización por ascenso freático, como ocurre en diferentes zonas agrícolas del Chaco y Espinal (Santoni et al. 2010; Amdan et al. 2013; Contreras et al. 2013). Sin embargo, la menor capacidad de las pasturas respecto de los bosques para usar el agua de precipitación sugiere que éstas podrían presentar pulsos de drenaje profundo si se registraran eventos de precipitación más grandes, intensos o frecuentes que los registrados en los 20 años transcurridos desde el cambio de cobertura. Por lo pronto, lo más esperable es que los cambios en el balance de agua introducidos 
por el reemplazo de bosques por pasturas se encuentren restringidos al resto de los flujos (intercepción, escurrimiento y evaporación).

A partir de varios de los resultados obtenidos, se estimó en forma aproximada la magnitud anual que representan los flujos de intercepción, escurrimiento y evaporación directa en el bosque y en las pasturas. Para ello se asumió un umbral fijo de "descuento" de agua por evento de precipitación para la intercepción (1.50 vs. $0.35 \mathrm{~mm}$ / evento; bosque y pastura, respectivamente) y la evaporación directa (1.2-4.4 vs. $7.0 \mathrm{~mm} /$ evento; bosque y pastura, respectivamente). Usando la lógica de "precipitación efectiva" (Zhang et al. 2001; Bates et al. 2006), se consideró que todos los eventos menores a dichos umbrales se perdieron en su totalidad y que los eventos mayores perdieron exactamente esa cantidad. Al aplicar estos criterios a la serie de datos de precipitación obtenida (Figura 2) se encontró que: i) la fracción de intercepción directa resultó menor en las pasturas respecto del bosque (3 vs. 11\%), ii) la fracción de escurrimiento superficial resultó apreciable en las pasturas (5-9\%, asumiendo que sólo se generó en los dos eventos más grandes/ intensos) y nula en el bosque, y iii) la fracción de evaporación directa aumentó fuertemente en las pasturas (40 vs. 9-28\%). Bajo estos supuestos, las pasturas tendrían disponible para la transpiración aproximadamente $20 \%$ menos de la lámina de agua precipitada que los bosques ( $\sim 50$ vs. $\sim 70 \%$ para la pastura y el bosque, respectivamente).

La evapotranspiración de los ecosistemas con precipitaciones entre 350 y $800 \mathrm{~mm} /$ año es muy sensible a los cambios en el tipo de cobertura vegetal dado que por debajo de $350 \mathrm{~mm} /$ año prácticamente todo lo precipitado es evapotranspirado, y por encima de $800 \mathrm{~mm} /$ año casi siempre existe una fracción de escurrimiento y drenaje profundo (Zhang et al. 2001; Huxman et al. 2005; Viglizzo et al. 2014). Dentro de ese rango de precipitaciones, una fracción muy grande de los sistemas terrestres se encuentra destinada a la producción ganadera y, por ende, están sujetos a cambios constantes de la cobertura vegetal como consecuencia de su reemplazo intencional o por la presión del ganado (Schlesinger et al. 1990; Asner et al. 2004; Bisigato et al. 2009). Los cambios más frecuentes son la reducción intencional de la vegetación leñosa, con el objetivo de aumentar la receptividad del sistema, o su incremento no intencional, producto de la arbustización por sobre-pastoreo (Van Auken 2000; Huxman et al. 2005; Wilcox and Huang 2010). El Chaco Árido representa un ejemplo del primer caso, donde la vegetación leñosa es suprimida de manera brusca y masiva para implantar pasturas. Sin embargo, en la última década comenzó a ser cada vez más frecuente la práctica del rolado, que elimina la vegetación leñosa arbustiva pero mantiene los ejemplares arbóreos de mediano y mayor porte (Kunst et al. 2003; Anriquez et al. 2005; Marchesini 2011). Los resultados de este trabajo, basados en mediciones de campo puntuales en el espacio y el tiempo, sugieren que los rolados, desde el punto de vista de la producción y de la partición de los flujos de agua, se encontrarían en una situación intermedia entre el bosque y la pastura. Esto se debe a que, por un lado, aumentan la receptividad ganadera del sistema (Blanco et al. 2005a), y por el otro, disminuyen los impactos sobre la evaporación potencial y el escurrimiento superficial.

\section{CONCLUSIONES}

Este trabajo mostró cómo el reemplazo de bosques secos nativos por pasturas alteró la partición de flujos de agua del Chaco Árido. Por un lado, disminuyó la fracción de la precipitación potencialmente disponible para transpiración, como producto de aumentos de la evaporación potencial del suelo y del escurrimiento superficial. Por otro lado, aumentó el riesgo potencial de erosión hídrica y la posibilidad de cosecha de agua en represas gracias al escurrimiento superficial en eventos extremos. Finalmente, las pasturas presentaron una captura de agua de lluvia espacialmente más homogénea que el bosque, producto de una menor redistribución de agua del canopeo y de la superficie del suelo. Este trabajo resalta la importancia del estudio de la dinámica del agua a escala de parche en paisajes semiáridos de baja pendiente regional, y ayuda a comprender cómo la interacción de las precipitaciones con la vegetación afecta el funcionamiento ecohidrológico del ecosistema.

Agradecimientos. Agradecemos a Francisco Murray, Javier Houspanossian, Eva L. Florio y Ulises Marconato por su colaboración en muchas de las mediciones de campo, a Marisa Puente por su apoyo logístico y a Fabián Scholz y a un revisor anónimo por mejorar la calidad y claridad de este manuscrito. Jorge L. Mercau, José Paruelo y Gervasio Piñeiro aportaron ideas importantes. Agradecemos también a Yeye Darvich y Ser Beef S.A. (especialmente 
a Nicolás Ríos Centeno, Alejandro Sánchez y Martín Ibarra) por permitirnos trabajar en sus establecimientos y proporcionarnos datos. Este trabajo se llevó adelante con el apoyo del International Development Research Center (IDRC, Canada 106601-001); el Inter-American
Institute for Global Change Research (IAI, SGP-CRA 2031 y CRN 3095, apoyado por la U.S. National Science Foundation, Grant GEO0452325), la Universidad de Buenos Aires (UBACyT 20020100100736), y la ANPCyT (PICT 2014-2790).

\section{REFERENCIAS}

Amdan, M., R. Aragón, E. G. Jobbágy, J. Volante, and J. M. Paruelo. 2013. Onset of deep drainage and salt mobilization following forest clearing and cultivation in the Chaco plains (Argentina). Water Resources Research 49:6601-6612.

Anriquez, A., A. Albanesi, C. Kunst, R. Ledesma, C. López, A. Rodríguez Torresi, and J. Godoy. 2005. Rolado de fachinales y calidad de suelos en el Chaco occidental, Argentina. Ciencia Del Suelo 23:145-157.

Asner, G. P., A. J. Elmore, L. P. Olander, R. E. Martin, and T. Harris. 2004. Grazing systems, ecosystem responses, and global change. Annual Review of Environmental Resources 29:261-299.

Austin, A. T., L. Yahdjian, J. M. Stark, J. Belnap, A. Porporato, U. Norton, D. A. Ravetta, and S. M. Schaeffer. 2004. Water pulses and biogeochemical cycles in arid and semiarid ecosystems. Oecologia 141:221-235.

Baldi, G., J. Houspanossian, F. Murray, A. A. Rosales, C. V. Rueda, and E. G. Jobbágy. 2015. Cultivating the dry forests of South America: Diversity of land users and imprints on ecosystem functioning. Journal of Arid Environments 123, 47-59.

Bates, J. D., T. Svejcar, R. F. Miller, and R. A. Angell. 2006. The effects of precipitation timing on sagebrush steppe vegetation. Journal of Arid Environments 64:670-697.

Bautista, S., Á. G. Mayor, J. Bourakhouadar, and J. Bellot. 2007. Plant spatial pattern predicts hillslope runoff and erosion in a semiarid mediterranean landscape. Ecosystems 10:987-998.

Bisigato, A. J., P. E. Villagra, J. O. Ares, and B. E. Rossi. 2009. Vegetation heterogeneity in Monte Desert ecosystems: A multi-scale approach linking patterns and processes. Journal of Arid Environments 73:182-191.

Blanco, L., F. Biurrun, and C. Ferrando. 2005a. Niveles de degradación de la vegetación del Chaco Arido. Una Aproximación cuantitativa a partir de imágenes satelitales. INTA. Serie de Publicaciones de Investigación EEA La Rioja, Argentina. Pp. 12.

Blanco, L. J., C. A. Ferrando, F. N. Biurrun, E. L. Orionte, P. Namur, D. J. Recalde, and G. D. Berone. 2005b. Vegetation responses to roller chopping and buffelgrass seeding in Argentina. Rangeland Ecology And Management 58:219224.

Bondeau, A., P. C. Smith, S. Zaehle, S. Schaphoff, W. Lucht, W. Cramer, D. Gerten, H. Lotze-Campen, C. Müller, M. Reichstein, and B. Smith. 2007. Modelling the role of agriculture for the 20th century global terrestrial carbon balance. Global Change Biology 13:679-706.

Breshears, D. D. and F. J. Barnes. 1999. Interrelationships between plant functional types and soil moisture heterogeneity for semiarid landscapes within the grassland/forest continuum: A unified conceptual model. Landscape Ecology 14:465-478.

Caldwell, T. G., M. H. Young, E. V. McDonald, and J. Zhu. 2012. Soil heterogeneity in Mojave Desert shrublands: Biotic and abiotic processes. Water Resources Research 48:W09551, doi:10.1029/2012WR011963.

Caldwell, T. G., M. H. Young, J. Zhu, and E. V. McDonald. 2008. Spatial structure of hydraulic properties from canopy to interspace in the Mojave desert. Geophysical Research Letters 35:L19406, doi:10.1029/2008GL035095.

Carlyle-Moses, D. E. 2004. Throughfall, stemflow, and canopy interception loss fluxes in a semi-arid Sierra Madre Oriental matorral community. Journal of Arid Environments 58:181-202.

Colman, E. 1947. A laboratory procdure for determining the field capacity of soils. Soil Science 63:277-284.

Connolly, R. D. 1998. Modelling effects of soil structure on the water balance of soil-crop systems: A review. Soil And Tillage Research 48:1-19.

Contreras, S., C. S. Santoni, and E. G. Jobbágy. 2013. Abrupt watercourse formation in a semiarid sedimentary landscape of central Argentina: The roles of forest clearing, rainfall variability and seismic activity. Ecohydrology 6:794-805.

Chapin, F. S., P. Matson, and H. A. Mooney. 2002. Terrestrial water y Energy Balance. Springer-Verlag, New York.

Del Grosso, S., W. Parton, T. Stohlgren, D. Zheng, D. Bachelet, S. Prince, K. Hibbard, and R. Olson. 2008. Global potential net primary production predicted from vegetation class, precipitation, and temperature. Ecology 89:2117-2126.

Eberbach, P. L. 2003. The eco-hydrology of partly cleared, native ecosystems in southern Australia: A review. Plant And Soil 257:357-369.

Farley, K. A., E. G. Jobbágy, and R. B. Jackson. 2005. Effects of afforestation on water yield: A global synthesis with implications for policy. Global Change Biology 11:1565-1576.

Foley, J. A., R. DeFries, G. P. Asner, C. Barford, G. Bonan, S. R. Carpenter, F. S. Chapin, M. T. Coe, G. C. Daily, H. K. Gibbs, J. H. Helkowski, T. Holloway, E. A. Howard, C. J. Kucharik, C. Monfreda, J. A. Patz, I. C. Prentice, N. Ramankutty, and P. K. Snyder. 2005. Global consequences of land use. Science 309:570-574.

Hao, Y., X. Kang, X. Wu, X. Cui, W. Liu, H. Zhang, Y. Li, Y. Wang, Z. Xu, and H. Zhao. 2013. Is frequency or amount of precipitation more important in controlling $\mathrm{CO} 2$ fluxes in the 30-year-old fenced and the moderately grazed temperate steppe? Agriculture, Ecosystems And Environment 171:63-71. 
Harper, C. W., J. M. Blair, P. A. Fay, A. K. Knapp, and J. D. Carlisle. 2005. Increased rainfall variability and reduced rainfall amount decreases soil CO2 flux in a grassland ecosystem. Global Change Biology 11:322-334.

Hillel, D. 1998. Environmental soil physics: Fundamentals, applications, and environmental considerations. Academic Press, United States of America. Pp. 771.

Huxman, T. E., M. D. Smith, P. A. Fay, A. K. Knapp, M. R. Shaw, M. E. Lolk, S. D. Smith, D. T. Tissue, J. C. Zak, J. F. Weltzin, W. T. Pockman, O. E. Sala, B. M. Haddad, J. Harte, G. W. Koch, S. Schwinning, E. E. Small, and D. G. Williams. 2004. Convergence across biomes to a common rain-use efficiency. Nature 429:651-654.

Huxman, T. E., B. P. Wilcox, D. D. Breshears, R. L. Scott, K. A. Snyder, E. E. Small, K. Hultine, W. T. Pockman, and R. B. Jackson. 2005. Ecohydrological implications of woody plant encroachment. Ecology 86:308-319.

Iriondo, M. 1993. Geomorphology and late quaternary of the Chaco (South America). Geomorphology 7:289-303.

Jobbágy, E. G., M. D. Nosetto, C. S. Santoni, and G. Baldi. 2008. El desafío ecohidrológico de las transiciones entre sistemas leñosos y herbáceos en la llanura chaco-pampeana. Ecología Austral 18:305-322.

Kleb, H. R. and S. D. Wilson. 1997. Vegetation effects on soil resource heterogeneity in prairie and forest. The American Naturalist 150:283-298.

Knapp, A. K., P. A. Fay, J. M. Blair, S. L. Collins, M. D. Smith, J. D. Carlisle, C. W. Harper, B. T. Danner, M. S. Lett, and J. K. McCarron. 2002. Rainfall variability, carbon cycling, and plant species diversity in a mesic grassland. Science 298:2202-2205.

Kunst, C., R. Ledesma, S. Bravo, A. Albanesi, A. Anriquez, H. Van Meer, and J. Godoy. 2012. Disrupting woody steady states in the Chaco region (Argentina): responses to combined disturbance treatments. Ecological Engineering $\mathbf{4 2}$ : $42-53$.

Kunst, C., R., M. Ledesma, G. Basan Nickish, D. Angella, P. J., and Godoy. 2003. Rolado de "fachinales" e infiltración de agua en el Chaco occidental (Argentina). Revista de Investigaciones Agropecuarias, 32:105-126.

Levia, D. F. and E. E. Frost. 2003. A review and evaluation of stemflow literature in the hydrologic and biogeochemical cycles of forested and agricultural ecosystems. Journal of Hydrology 274:1-29.

Ludwig, J. A., B. P. Wilcox, D. D. Breshears, D. J. Tongway, and A. C. Imeson. 2005. Vegetation patches and runofferosion as interacting ecohydrological processes in semiarid landscapes. Ecology 86:288-297.

Llorens, P. and F. Domingo. 2007. Rainfall partitioning by vegetation under Mediterranean conditions. A review of studies in Europe. Journal of Hydrology 335:37-54.

Magliano, P. N., D. D. Breshears, R. J. Fernández, and E. G. Jobbágy. 2015a. Rainfall intensity switches ecohydrological runoff/runon redistribution patterns in dryland vegetation patches. Ecological Applications 25:2094-2100.

Magliano, P. N., R. J. Fernández, J. L. Mercau, and E. G. Jobbágy. 2015b. Precipitation event distribution in central argentina: Spatial and temporal patterns. Ecohydrology 8:94-104.

Magliano, P. N., F. Murray, G. Baldi, S. Aurand, R. A. Páez, W. Harder, and E. G. Jobbágy. 2015c. Rainwater harvesting in Dry Chaco: Regional distribution and local water balance. Journal of Arid Environments 123:93-102.

Marchesini, V. 2011. Cambios en el uso de la tierra y el balance de agua en ecosistemas semiáridos: el desmonte selectivo en el Chaco árido analizado a diferentes escalas espaciales. Tesis Doctoral. Universidad de Buenos Aires, Buenos Aires, Argentina.

Marchesini, V. A., R. J. Fernández, and E. G. Jobbágy. 2013. Salt leaching leads to drier soils in disturbed semiarid woodlands of central Argentina. Oecologia 171:1003-1012.

Marchesini, V. A., R. J. Fernández, J. F. Reynolds, J. A. Sobrino, and C. M. Di Bella. 2015. Changes in evapotranspiration and phenology as consequences of shrub removal in dry forests of central Argentina. Ecohydrology 8:1304-1311.

Martinez-Meza, E. and W. G. Whitford. 1996. Stemflow, throughfall and channelization of stemflow by roots in three Chihuahuan desert shrubs. Journal of Arid Environments 32:271-287.

Morello, J., and J. Adámoli. 1974. Las grandes unidades de vegetación y ambiente del Chaco argentino. Segunda Parte: Vegetación y ambiente de la Provincia del Chaco. INTA Serie fitogeográfica 13.

Morello, J., and C. S. Toledo. 1959. El bosque chaqueño I. Paisaje primitivo, paisaje natural y paisaje cultural en el oriente de Salta. Revista Agronómica del Noroeste Argentino 3:208.

Newman, B. D., B. P. Wilcox, S. R. Archer, D. D. Breshears, C. N. Dahm, C. J. Duffy, N. G. McDowell, F. M. Phillips, B. R. Scanlon, and E. R. Vivoni. 2006. Ecohydrology of water-limited environments: A scientific vision. Water Resources Research 42:W06302, doi:10.1029/2005WR004141.

Nicholson, S. E. 2011. Precipitation in the drylands. Dryland climatology. Cambridge University Press.

Nosetto, M. D., E. G. Jobbágy, A. B. Brizuela, and R. B. Jackson. 2012. The hydrologic consequences of land cover change in central Argentina. Agriculture, Ecosystems And Environment 154:2-11.

Noy-Meir, I. 1973. Desert ecosystems, environment, and producers. Annual Review of Ecological Systems 4:25-32.

Oweis, T., and A. Hachum. 2009. Water Harvesting for Improved Rainfed Agriculture in the Dry Environments. Pp. 164-181 in S. P. Wani, editor. Rainfed Agriculture: Unlocking the Potential. CAB International.

Pennington, T. R., D. E. Prado, and C. A. Pendry. 2000. Neotropical seasonally dry forests and Quaternary vegetation changes. Journal of Biogeography 27:261-273.

Peña Zubiate, C. A., D. L. Anderson, M. A. Demmi, J. L. Saenz, and A. D’Hiriart. 1998. Carta de suelos y vegetación de la provincia de San Luis. Instituto Nacional de Tecnología Agropecuaria, San Luis, Argentina.

Reid, K. D., B. P. Wilcox, D. D. Breshears, and L. MacDonald. 1999. Runoff and erosion in a pinon-juniper woodland: Influence of vegetation patches. Soil Science Society of America Journal 63:1869-1879. 
Reynolds, J. F., R. A. Virginia, P. R. Kemp, A. G. De Soyza, and D. C. Tremmel. 1999. Impact of drought on desert shrubs: Effects of seasonality and degree of resource island development. Ecological Monographs 69:69-106.

Rueda, C. V., G. Baldi, S. R. Verón, and E. G. Jobbágy. 2013. Apropiación humana de la producción primaria en el Chaco Seco. Ecologia Austral 23:44-54.

Sala, O. E., W. J. Parton, L. A. Joyce, and W. K. Lauenroth. 1988. Primary production of the central grassland region of the United States. Ecology 69:40-45.

Santoni, C. S., E. G. Jobbagy, and S. Contreras. 2010. Vadose zone transport in dry forests of central Argentina: role of land use. Water Resources Research 46:W10541.

Scanlon, B. R. 1991. Evaluation of moisture flux from chloride data in desert soils. Journal of Hydrology 128:137-156.

Scanlon, B. R., R. W. Healy, and P. G. Cook. 2002. Choosing appropriate techniques for quantifying groundwater recharge. Hydrogeology Journal 10:18-39.

Scanlon, B. R., R. C. Reedy, D. A. Stonestrom, D. E. Prudic, and K. F. Dennehy. 2005. Impact of land use and land cover change on groundwater recharge and quality in the southwestern US. Global Change Biology 11:1577-1593.

Schimel, D. S. 2010. Drylands in the earth system. Science 327:418-419.

Schlesinger, W. H., and S. Jasechko. 2014. Transpiration in the global water cycle. Agricultural And Forest Meteorology 189-190:115-117.

Schlesinger, W. H., J. F. Reynolds, G. L. Cunningham, L. F. Huenneke, W. M. Jarrell, R. A. Virginia, and W. G. Whitford. 1990. Biological feedbacks in global desertification. Science 247:1043-1048.

Schwinning, S., O. E. Sala, M. E. Loik, and J. R. Ehleringer. 2004. Thresholds, memory, and seasonality: Understanding pulse dynamics in arid/semi-arid ecosystems. Oecologia 141:191-193.

Van Auken, O. W. 2000. Shrub invasions of North American semiarid grasslands. Annual Review of Ecology And Systematics 31:197-215.

Viglizzo, E. F., M. D. Nosetto, E. G. Jobbágy, M. F. Ricard, and F. C. Frank. 2014. The ecohydrology of ecosystem transitions: a meta analysis. Ecohydrology DOI:10.1002/eco.1540.

Wang, Y., Y. Li, X. Ye, Y. Chu, and X. Wang. 2010. Profile storage of organic/inorganic carbon in soil: From forest to desert. Science of The Total Environment 408:1925-1931.

Wilcox, B. P., and Y. Huang. 2010. Woody plant encroachment paradox: Rivers rebound as degraded grasslands convert to woodlands. Geophysical Research Letters 37:1-5.

Yahdjian, L., and O. E. Sala. 2006. Vegetation structure constrains primary production response to water availability in the patagonian steppe. Ecology 87:952-962.

Yaseef, N. R., D. Yakir, E. Rotenberg, G. Schiller, and S. Cohen. 2010. Ecohydrology of a semi-arid forest: Partitioning among water balance components and its implications for predicted precipitation changes. Ecohydrology 3:143154.

Zhang, L., W. R. Dawes, and G. R. Walker. 2001. Response of mean annual evapotranspiration to vegetation changes at catchment scale. Water Resources Research 37:701-708. 